\title{
Métabolisme des rétinoïdes et cancer
}

> Les rétinoïdes jouent des rôles importants dans la différenciation cellulaire et l'apoptose, notamMaxime Parisotto, Hélène Brodeur, Pangala V. Bhat, Sylvie Mader ment dans les tissus épithéliaux. L'emploi d'acide rétinoïque $(A R)$ dans le traitement de la leucémie promyélocytique aiguë ( $A P L)$ a fourni la première démonstration de succès d'une thérapie anticancéreuse par un rétinoïde. Depuis, des traitements à base de rétinoïdes se sont révélés être d'une efficacité variable selon le type de cancer. Les voies métaboliques de synthèse et d'inactivation de l'AR sont affectées lors de la tumorigenèse et du traitement par les rétinoïdes. Une meilleure compréhension de la modulation de l'expression ou de l'activité des enzymes du métabolisme de I'AR pourrait permettre d'optimiser l'utilisation des rétinoïdes dans le traitement de différents types de cancers. <

L'acide rétinoïque (AR), qui est synthétisé dans les tissus par deux étapes successives d'oxydation à partir du rétinol (vitamine A) provenant de l'alimentation, est la principale forme active de rétinoïde avec le rétinal 11cis, qui participe au cycle visuel. Deux isomères de l'AR, les formes tout-trans et 9-cis (Figure I), sont des ligands de deux familles distinctes de récepteurs nucléaires, les RAR, qui interagissent avec les deux formes d'AR, et les RXR, qui s'associent spécifiquement à l'AR 9-cis (Figure 1). Il est à noter cependant que l'existence de l'AR 9-cis in vivo reste controversée [1]. Un autre isomère naturel, I'AR 13-cis, ne lie pas ces récepteurs directement mais après isomérisation en AR tout-trans [2]. Les récepteurs de l'AR agissent comme facteurs de transcription, s'associant sous forme d'homodimères de RXR ou d'hétérodimères RAR-RXR à des séquences spécifiques d'ADN. Ces séquences sont composées de motifs répétés séparés par 1, 2, ou 5 paires de bases (Figure 1). La complexité combinatoire des hétérodimères provenant de l'existence de plusieurs formes de RAR et de RXR (trois gènes $\alpha$, $\beta$ et $\gamma$, et plusieurs isoformes pour chaque gène) assure une régulation transcriptionnelle complexe des gènes cibles par l'AR (Figure 1) [1, 3, 4]. De plus, les RXR peuvent également former des hétérodimères avec

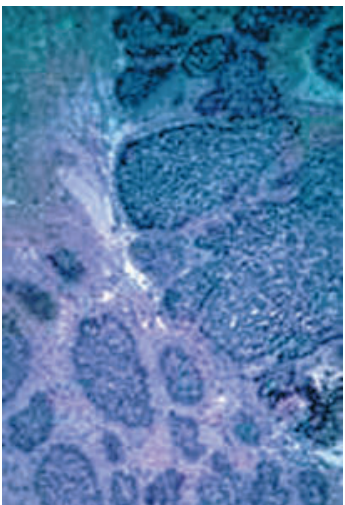

M. Parisotto, S. Mader :

Département de biochimie et Institut de recherche en immunologie et cancérologie, Université de Montréal, CP 6128, succursale Centre-ville, Montréal (Québec), H3C 3J7 Canada. Sylvie.Mader@umontreal.ca H. Brodeur, P.V. Bhat : Laboratoire de nutrition et cancer, Centre

d'autres récepteurs hospitalier de l'Université nucléaires, dont cer- de Montréal-Hôtel Dieu, tains (PPAR, FXR, LXR, Département de Médecine, Nurrl) sont permissifs Université de Montréal, CP 6128, pourune réponse trans- succursale Centre-ville, Montréal criptionnelle à I'AR 9- (Québec), H3C 3J7 Canada. cis [5]. La réponse d'un tissu à l'AR est gouvernée en partie par les profils d'expression des différents types de récepteurs [3, 4], mais aussi par ceux des enzymes impliquées dans le métabolisme de l'AR.

\section{Rôle de l'acide rétinoïque dans la différenciation cellulaire et le cancer}

Le rôle important des rétinoïdes dans la différenciation cellulaire et le cancer a été démontré par les effets d'une carence ou d'un excès de rétinoïdes dans la diète, ainsi que par des altérations expérimentales ou pathologiques de l'expression des récepteurs. La déficience en vitamine $A$ comme la délétion combinée de deux ou trois RAR provoquent de graves anomalies du développement chez la souris, avec des malformations craniofaciales, rénales et squelettiques, démontrant le rôle important de ces récepteurs dans la médiation de l'action de l'AR en tant qu'agent de différenciation $[3,4]$. Chez l'adulte, la déficience en vitamine $A$ a été associée à une incidence accrue de cancers et à une

Article reçu le 15 septembre 2005, accepté le 30 mars 2006. 
plus grande sensibilité aux carcinogènes chimiques, tandis que des rétinoïdes naturels ou synthétiques inhibent la tumorigenèse induite par des carcinogènes au niveau de différents tissus [6-9]. Enfin, la diminution de l'expression du récepteur RAR- $\beta 2$ par l'expression de transgènes antisens a pour conséquence le développement de tumeurs pulmonaires chez la souris [10].

Divers rétinoïdes sont utilisés en clinique dans des approches de chimioprévention pour des patients porteurs de lésions pré-malignes telles la kératinose actinique, leukoplakie ou dysplasie cervicale, ou après le traitement de tumeurs primaires dans le cancer de la peau, des voies orales, du poumon ou du sein [7-9]. L'AR tout-trans et 13-cis sont actuellement utilisés pour le traitement de la leucémie promyélocytique aiguë $(\mathrm{APL})$, du cancer de la prostate et du neuroblastome $[2,8,9]$. L'APL implique des translocations chromosomiques caractéristiques affectant RAR $\alpha$ (le plus fréquemment avec le gène PML, mais aussi avec PLZF, NPM, NuMa, STAT5b [11]). L'administration d'AR tout-trans permet la différenciation des promyélocytes contenant la fusion PML/RAR $\alpha$ et induit des rémissions complètes mais transitoires. L'AR 13-cis présente des propriétés pharmacocinétiques plus favorables que l'AR tout-trans, avec des concentrations plasmatiques plus élevées et une demi-vie plus longue, et pourrait donc être plus efficace dans le traitement de tumeurs, particulièrement lorsque administré de manière intermittente afin de minimiser l'induction d'enzymes d'inactivation [2]. Les «rexinoïdes » ou ligands sélectifs pour les RXR (par exemple le bexarotène, qui a démontré des résultats encourageants pour le traitement du cancer du poumon en combinaison avec la chimiothérapie) agiraient pour leur part en modulant les voies de signalisation d'autres récepteurs nucléaires par l'intermédiaire d'héterodimères impliquant les RXR $[4,8,9,12]$.

L'utilisation de rétinoïdes en clinique est cependant limitée par le développement d'une résistance intrinsèque lors de la carcinogenèse dans les tumeurs solides et d'une résistance acquise lors du traitement de l'APL par I'AR tout-trans. Des mutations dominantes négatives surviennent dans la fusion PML/RAR $[6,9]$. Une inactivation épigénétique des RAR (RAR $\beta$ en particulier) a également été mise en évidence dans de nombreuses tumeurs solides, et peut être abolie par traitement aux inhibiteurs de méthylation de l'ADN [9]. De plus, plusieurs mécanismes peuvent contribuer à une résistance aux rétinoïdes en limitant les concentrations d'AR intracellulaire, incluant un rôle possible mais controversé de la protéine de résistance aux drogues multiples, et l'induction de l'expression d'enzymes d'inactivation de I'AR [7-9]. La résistance à l'AR pourrait donc être combattue par l'utilisation d'inhibiteurs du métabolisme de I'AR ou de rétinoïdes synthétiques non soumis à ces processus d'inactivation.

\section{Synthèse et dégradation de l'acide rétinoïque}

Le rétinol est le rétinoïde majoritaire dans le sang, provenant de la conversion du $\beta$-carotène et des rétinyl esters alimentaires dans l'intestin et/ou des rétinyl esters stockés dans le foie. L'AR est présent en beaucoup plus faibles concentrations dans le plasma, et est synthétisé dans les tissus possédant le système enzymatique approprié. L'oxydation du rétinol en $A R$ est un processus enzymatique en deux étapes dont le rétinal est le métabolite intermédiaire, la première oxydation étant réversible et la deuxième irréversible. La synthèse de I'AR implique plusieurs déshydrogénases appartenant à 4 familles distinctes, soient les alcool déshydrogénases $(A D H)$, les déshydrogénases/réductases à chaînes courtes (SDR), les aldéhyde déshydrogéna-

Figure 1. Activation de récepteurs nucléaires par l'acide rétinoïque. 
ses (ALDH), ainsi que les enzymes de la famille des cytochromes P450 (Figure 2) [1, 13]. Certaines de ces enzymes montrent une sélectivité envers les isomères du rétinol(Rol) et/ou rétinal(Ral) (Tableau l). L'importance des ADH et des ALDH dans la synthèse de I'AR lors de l'embryogenèse et chez l'adulte est illustrée par leurs domaines d'expression et par les phénotypes résultant de l'inactivation génique chez la souris. ADH3 est exprimée de manière ubiquitaire lors de l'embryogenèse, assurant une conversion du rétinol en rétinal dans la plupart

\begin{tabular}{|c|c|c|c|c|}
\hline $\begin{array}{l}\text { Enzymes } \\
\text { métaboliques }\end{array}$ & tout-trans & 9-cis & 13 -cis & Références \\
\hline \multicolumn{5}{|l|}{ Famille ADH } \\
\hline $\mathrm{ADHl}$ & $+($ Rol/Ral $)$ & $+($ Rol/Ral $)$ & - & {$[34,35]$} \\
\hline $\mathrm{ADH} 3$ & $+(\operatorname{Rol})$ & $?$ & $?$ & {$[36-39]$} \\
\hline $\mathrm{ADH} 4$ & $+(\mathrm{Rol} / \mathrm{Ral})$ & $+(\mathrm{Rol} / \mathrm{Ral})$ & $+(\mathrm{Rol} / \mathrm{Ral})$ & {$[34,39,40]$} \\
\hline \multicolumn{5}{|l|}{ Famille RALDH } \\
\hline RALDHI & $+(\mathrm{Ral})$ & $+(\mathrm{Ral})$ & $+\star(\mathrm{Ral})$ & [41-44] \\
\hline RALDH2 & $+(\mathrm{Ral})$ & $+(\mathrm{Ral})$ & $+(\mathrm{Ral})$ & {$[45]$} \\
\hline RALDH3 & $+(\mathrm{Ral})$ & $?$ & ? & {$[28,46]$} \\
\hline RALDH4 & - & $+(\mathrm{Ral})$ & $+/-(\mathrm{Ral})$ & {$[47,48]$} \\
\hline \multicolumn{5}{|l|}{ Famille CyP26 } \\
\hline сур26Al & $+(A R)$ & $-(A R)$ & $-(A R)$ & {$[49,50]$} \\
\hline Сур26Bl & $+(A R)$ & $-(A R)$ & $-(A R)$ & {$[51]$} \\
\hline Сур26Cl & $+(A R)$ & $+(A R)$ & $-(A R)$ & {$[51]$} \\
\hline $\begin{array}{l}\text { Protéines } \\
\text { de liaison }\end{array}$ & tout-trans & 9-cis & 13-cis & Références \\
\hline \multicolumn{5}{|l|}{ CRBP } \\
\hline CRBPI & $+(\operatorname{Rol})$ & - (Rol) & $+($ Rol $)$ & [52-54] \\
\hline CRBPII & $+(\mathrm{Rol} / \mathrm{Ral})$ & - (Rol) & $+($ Rol $)$ & {$[54-56]$} \\
\hline CRBPIII & $+(\operatorname{Rol})$ & $+(\mathrm{Rol})$ & $+($ Rol $)$ & [18] \\
\hline \multicolumn{5}{|l|}{ CRABP } \\
\hline CRABPI & $+(A R)$ & - & - & [57-62] \\
\hline CRABPII & $+(A R)$ & - & - & {$[58,59,61,62]$} \\
\hline Récepteurs & tout-trans & 9-cis & 13-cis & Références \\
\hline RAR & $+(A R)$ & $+(A R)$ & - & {$[59,63]$} \\
\hline RXR & - & $+(A R)$ & - & {$[59,64]$} \\
\hline
\end{tabular}

Tableau I. Spécificité de substrat des enzymes du métabolisme de l'AR.

* Variations entre espèces. des tissus. RALDH2 est principalement responsable de la conversion histo-spécifique du rétinal en $A R$ dans le mésoderme embryonnaire, la suppression ciblée de RALDH2 chez la souris provoquant une mort embryonnaire précoce [13]. Chez l'adulte, les ADH de classe I et IV ainsi que RALDHI/4 sont exprimées dans les épithéliums qui dépendent de I'AR pour leur différenciation normale [13]. Le rôle exact dans la biosynthèse de l'AR des membres de la famille des SDR et des P450, ainsi que d'autres enzymes telles l'aldéhyde oxydase et la xanthine oxydase, nécessite par contre plus de données sur leurs domaines d'expression et leur spécificité de substrat in vivo. Certaines des enzymes SDR ont en effet une activité supérieure sur d'autres substrats, tels les stéroïdes ou prostanoïdes, ou participent à la synthèse de 11 -cis rétinal pour le cycle visuel $[1,13]$.

Les AR tout-trans, 9-cis et 13-cis sont convertis en dérivés inactifs, la première étape étant une conversion en 4-hydroxy- et 18-hydroxyAR (Figure 2). La conversion en 4-hydroxy-AR dépend de l'action d'enzymes P450, dont les plus spécifiques et efficaces sont les CYP26Al/Bl/Cl, qui ont comme substrat l'AR tout-trans, CYP26Cl métabolisant également I'AR 9-cis [7, 2] (Tableau I). L'ablation génétique de CYP26Al et de CYP26Bl révèle des anomalies de développement ressemblant fortement aux effets tératogènes de l'AR touttrans [14, 15], Chez les mutants CyP26Al nuls, la suppression hétérozygote de RALDH2 ou l'ablation de RARy corrige le phénotype d'excès d'AR et prolonge la survie embryonnaire $[16,17]$, suggérant que le rôle clé de CyP26Al est de maintenir des régions de l'embryon dans un état dépourvu d'AR. L'expression de CYP26Al est inductible par l'AR dans le foie et plusieurs tissus extra-hépatiques, résultant en une boucle de rétroaction négative sur les niveaux d'AR tout-trans [7]. D'autres enzymes à cytochrome $\mathrm{P} 450$ pourraient inactiver les formes tout-trans, 9-cis ou 13-cis, mais ont des valeurs de $\mathrm{Km}$ beaucoup plus élevées $[1,2]$.

Deux familles de protéines cytoplasmiques, les cellular retinol-binding proteins (CRBP I, II et III) et les cellular RA-binding proteins (CRABP I et II) pourraient aussi participer au métabolisme de la vitamine $A$ [1-4, 18]. CRBPI n'est pas nécessaire au développement normal et à la survie pour autant que la diète ne soit pas carencée en vitamine $A$, mais joue un rôle important dans l'accumulation des rétinyl esters dans le foie [19]. CRABPI a été impliquée dans le catabolisme 
de l'AR et CRABPII dans la présentation de l'AR aux RAR, cependant l'inactivation génique de CRABPI et II chez la souris ne semble pas affecter la signalisation par l'AR de façon marquée [20].

\section{Séquestration et inactivation de l'AR lors du traitement anticancéreux}

L'AR induit l'expression de CRABPII dans les cellules hématopoïétiques normales et leucémiques; il a été proposé que CRABPII pourrait séquestrer I'AR tout-trans et ainsi contribuer à une résistance au traitement. Cependant, les données sur une corrélation entre l'expression accrue de CRABPII et la récurrence de I'APL sont contradictoires, et d'un autre côté un rôle de coactivateur des RARs a été proposé pour CRABPII $[2,9]$. D'un autre côté, l'induction de l'expression de CyP26Al par l'AR, susceptible de diminuer les concentrations d'AR dans les cellules leucémiques et/ou dans le sang, pourrait contribuer à limiter la sensibilité aux traitements par l'AR $[6,7,9]$.

Une stratégie alternative à la thérapie par I'AR tout-trans exogène est d'augmenter le taux d'AR circulant et/ou dans les tumeurs en inhibant les enzymes responsables de son inactivation par des retinoic acid metabolism blocking agents (RAMBA) [6]. In vivo, le traitement avec le RAMBA le plus étudié, le liarozole fumarate (LIA), qui augmente le taux d'AR dans le plasma et dans les tissus, a engendré des résultats prometteurs dans le traitement des cancers avancés du sein et de la prostate $[2,7]$. Toutefois, le LIA inhibe plusieurs enzymes P450, incluant l'aromatase, responsable de la biosynthèse des œstrogènes, et la 25-hydroxyvitamine D-24hydroxylase, responsable de l'inactivation de la $1,25-(\mathrm{OH})_{2} \mathrm{D} 3$, ce qui pourrait contribuer à ses propriétés anticancéreuses in vivo. De nouveaux RAMBA récemment développés, tels le R115866 [21] ou le R116010 [22] démontrent une plus grande efficacité et spécificité dans l'inhibition des CyP26. Par ailleurs, I'utilisation de rétinoïdes résistants au catabolisme, mais conservant une affinité élevée pour les récepteurs, pourrait aussi permettre d'augmenter l'efficacité du traitement anti-cancéreux.

\section{Déficience de l'absorption du rétinol et de la biosynthèse d'AR lors de la tumorigenèse}

De plus en plus de travaux démontrent une altération de l'absorption des rétinoïdes et de la biosynthèse de I'AR au niveau de la tumeur elle-même. II a été observé que la concentration en AR tout-trans est plus faible dans certains tissus cancéreux que dans les tissus normaux correspondants, comme par exemple dans le cas du cancer de la prostate [6]. Une diminution de l'absorption de rétinol et de l'activité rétinal oxydase a été observée dans un modèle de carcinome mammaire induit par la N-méthyl-Nnitroso-urée chez le rat, par rapport aux tissus sains [23]. Une autre étude a observé une diminution de la production de rétinyl esters et de la synthèse d'AR dans deux lignées de cancer du sein humaines [24]. Une inactivation épigénétique de CRBPI est observée dans plusieurs cancers, dont le cancer du sein, et la réexpression de cette protéine dans des cellules mammaires immortalisées supprime la tumorigenèse in vivo [25]. Une capacité fortement réduite de synthétiser I'AR a été observée dans plusieurs lignées humaines de carcinome mammaire même en présence de liarozole, démontrant que l'inactivation de I'AR par les enzymes P450 n'est pas responsable de la faible synthèse d'AR [26]. Les cellules humaines MCF-7 de cancer du sein transfectées avec I'ADNC de $\mathrm{CRDH}$ (RDH5) retrouvent la capacité d'absorber rapidement et d'oxyder le rétinol 9-cis en rétinal 9cis, mais pas en AR 9-cis [27]. En effet, les cellules MCF7 n'expriment pas la RALDH3 trouvée dans les cellules épithéliales mammaires normales [28].

D'autres déficiences dans les voies de biosynthèse de I'AR ont été observées dans divers types de cancer.

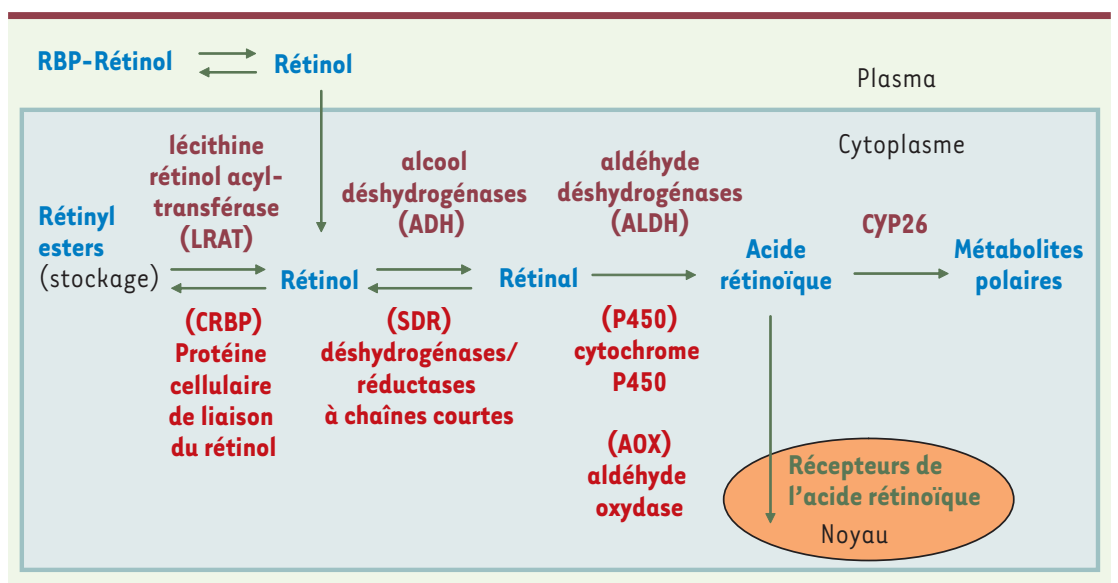

Figure 2. Étapes enzymatiques impliquées dans la biosynthèse de l'AR à partir du rétinol.
L'expression de RALDH2 est fréquemment réduite dans les tumeurs de la prostate, correspondant à une durée de rémission plus courte; le traitement par un inhibiteur de la méthylation de I'ADN permet de restaurer l'expression de ce gène dans la lignée cellulaire DU145 [29]. Dans les tumeurs du poumon l'expression de RALDHl est réduite d'un facteur 10 environ [30]. Dans les adénomes et carcinomes du côlon, une réduction de l'expression de deux gènes impliqués dans la biosynthèse de I'AR, RDH5 et RDHL (DHRS9) a été observée par rapport aux tissus normaux. La conversion du rétinol en AR était aussi 
plus faible dans 7 lignées de carcinome du côlon en comparaison avec des cellules normales [31]. Enfin, l'expression de la RoDH-4 est nettement réduite dans les tumeurs de l'endomètre [32] et le gène humain retSDRI est fréquemment perdu dans des cellules NB portant une amplification de NMYC [33]. Dans l'ensemble, ces observations suggèrent donc qu'une altération de l'expression d'enzymes essentielles à la biosynthèse d'AR in situ peut être associée au développement de plusieurs types de cancer. Les mécanismes de ces altérations restent à explorer, et pourraient impliquer une inactivation épigénétique des gènes correspondants ou de facteurs transcriptionnels contrôlant leur expression, dont potentiellement dans certains cas les RAR eux-mêmes.

\section{Conclusions}

L'inactivation métabolique inductible des rétinoïdes pourrait limiter l'utilisation thérapeutique de composés substrats de cette voie métabolique, conduisant au développement d'agents bloqueurs du métabolisme de I'AR. Cependant, la réduction de la production d'AR observée dans certains types de cancers pourrait limiter l'efficacité de ces composés et indiquer l'importance de tester ces inhibiteurs en combinaison avec l'administration d'AR plutôt qu'en thérapie simple, ou de développer des rétinoïdes résistants à l'inactivation métabolique. Finalement, quelle que soit l'approche retenue, la toxicité associée au traitement par les rétinoïdes reste un problème à résoudre. $\diamond$

\section{SUMMARY}

\section{Retinoid metabolism and cancer}

Retinoids play important roles in cell differentiation and apoptosis, notably in epithelial tissues. Their utility in cancer therapy has been demonstrated in specific cancer types. Use of retinoic acid (RA) in the treatment of acute promyelocytic leukemia was the first successful example of retinoid-based differentiation therapy. RA has since been evaluated for treatment of other cancers, revealing variable effectiveness. The observation that expression of enzymes involved in RA biosynthesis is suppressed during tumorigenesis suggests that intratumor depletion in RA levels may contribute to tumor development and argues for the use of retinoids in cancer treatment. However, the induction of RA-inactivating enzymes is one of the mechanisms that may limit the efficacy of retinoid therapy and contribute to acquired resistance to RA treatment, suggesting that retinoic acid metabolism blocking agents may be effective agents in differentiation therapy. $\diamond$

\section{RÉFÉRENCES}

1. Napoli JL. Interactions of retinoid binding proteins and enzymes in retinoid metabolism. Biochim Biophys Acta 1999; 1440 : 139-62.

2. Armstrong JL, Redfern CP, Veal GJ. 13-cis retinoic acid and isomerisation in paediatric oncology--is changing shape the key to success? Biochem Pharmacol 2005; 69: 1299-306.

3. Giguere V. Retinoic acid receptors and cellular retinoid binding proteins: complex interplay in retinoid signaling. Endocrinol Rev $1994 ; 15: 61-79$.

4. Chambon P. A decade of molecular biology of retinoic acid receptors. FASEB J 1996 ; $10: 940-54$.

5. Shulman Al, Larson C, Mangelsdorf DJ, et al. Structural determinants of allosteric ligand activation in RXR heterodimers. Cell 2004 ; $116: 417-29$.

6. Miller WH Jr. The emerging role of retinoids and retinoic acid metabolism blocking agents in the treatment of cancer. Cancer 1998; $83: 1471-82$.

7. Petkovich PM. Retinoic acid metabolism. J Am Acad Dermatol 2001 ; 45 : S136-42.

8. Sun SY, Lotan R. Retinoids and their receptors in cancer development and chemoprevention. Crit Rev Oncol Hematol $2002 ; 41: 41-55$.

9. Freemantle SJ, Spinella MJ, Dmitrovsky $\varepsilon$. Retinoids in cancer therapy and chemoprevention: promise meets resistance. Oncogene $2003 ; 22: 7305-15$.

10. Berard J, Laboune F, Mukuna M, et al. Lung tumors in mice expressing an antisense RARbeta2 transgene. FASEB J $1996 ; 10: 1091-7$.

11. Pitha-Rowe I, Petty WJ, Kitareewan S, et al. Retinoid target genes in acute promyelocytic leukemia. Leukemia 2003; $17:$ 1723-30.

12. Rigas JR, Dragnev KH. Emerging role of rexinoids in non-small cell lung cancer: focus on bexarotene. Oncologist 2005; $10: 22-33$.

13. Duester G, Mic FA, Molotkov A. Cytosolic retinoid dehydrogenases govern ubiquitous metabolism of retinol to retinaldehyde followed by tissue-specific metabolism to retinoic acid. Chem Biol Interact 2003; 143-144: 201-10.

14. Abu-Abed S, Dolle P, Metzger D, et al. The retinoic acid-metabolizing enzyme, CyP26Al, is essential for normal hindbrain patterning, vertebral identity, and development of posterior structures. Genes Dev $2001 ; 15: 226-40$.

15. Yashiro K, Zhao X, Uehara $M$, et al. Regulation of retinoic acid distribution is required for proximodistal patterning and outgrowth of the developing mouse limb. Dev Cell $2004 ; 6: 411-22$.

16. Niederreither K, Abu-Abed S, Schuhbaur B, et al. Genetic evidence that oxidative derivatives of retinoic acid are not involved in retinoid signaling during mouse development. Nat Genet $2002 ; 31: 84-8$.

17. Abu-Abed S, Dolle P, Metzger D, et al. Developing with lethal RA levels: genetic ablation of Rarg can restore the viability of mice lacking Cyp26al. Development $2003 ; 130$ : 1449-59.

18. Vogel S, Mendelsohn CL, Mertz JR, et al. Characterization of a new member of the fatty acidbinding protein family that binds all-trans-retinol. J Biol Chem $2001 ; 276$ : 1353-60.

19. Ghyselinck NB, Bavik C, Sapin V, et al. Cellular retinol-binding protein I is essential for vitamin A homeostasis. EMBO J $1999 ; 18: 4903-14$.

20. Lampron C, Rochette-Egly C, Gorry P, et al. Mice deficient in cellular retinoic acid binding protein II (CRABPII) or in both CRABPI and CRABPII are essentially normal. Development $1995 ; 121: 539-48$.

21. Stoppie $P$, Borgers $M$, Borghgraef $P$, et al. R115866 inhibits all-trans-retinoic acid metabolism and exerts retinoidal effects in rodents. J Pharmacol Exp Ther 2000 ; 293: 304-12.

22. Armstrong JL, Ruiz M, Boddy AV, et al. Increasing the intracellular availability of all-trans retinoic acid in neuroblastoma cells. BrJ Cancer 2005; $92: 696-704$.

23. Bhat PV, Lacroix A. Metabolism of retinol and retinoic acid in $\mathrm{N}$-methyl- $\mathrm{N}$-nitrosoureainduced mammary carcinomas in rats. Cancer Res $1989 ; 49: 139-44$.

24. Hayden LJ, Satre MA. Alterations in cellular retinol metabolism contribute to differential retinoid responsiveness in normal human mammary epithelial cells versus breast cancer cells. Breast Cancer Res Treat $2002 ; 72: 95-105$.

25. Farias EF, Ong DE, Ghyselinck NB, et al. Cellular retinol-binding protein I, a regulator of breast epithelial retinoic acid receptor activity, cell differentiation, and tumorigenicity. J Natl Cancer Inst $2005 ; 97: 21-9$.

26. Mira YLR, Zheng WL, Kuppumbatti YS, et al. Retinol conversion to retinoic acid is impaired in breast cancer cell lines relative to normal cells. J Cell Physiol $2000 ; 185: 302-9$.

27. Paik J, Blaner WS, Swisshelm K. Cis-retinol dehydrogenase: 9-cis-retinol metabolism and its effect on proliferation of human MCF7 breast cancer cells. Exp Cell Res $2005 ; 303$ : 183-96.

28. Rexer BN, Zheng WL, Ong DE. Retinoic acid biosynthesis by normal human breast epithelium is via aldehyde dehydrogenase 6, absent in MCF-7 cells. Cancer Res $2001 ; 61: 7065-70$.

29. Kim H, Lapointe J, Kaygusuz G, et al. The retinoic acid synthesis gene ALDHla2 is a candidate tumor suppressor in prostate cancer. Cancer Res $2005 ; 65: 8118-24$.

30. Dragani TA, Falvella FS, Manenti G, et al. Downexpression of aldehyde dehydrogenase 1 in murine lung tumors. Mol Carcinog 1996; $16: 123-5$.

31. Jette C, Peterson PW, Sandoval IT, et al. The tumor suppressor adenomatous polyposis coli and caudal related homeodomain protein regulate expression of retinol dehydrogenase L. J Biol Chem $2004 ; 279$ : 34397-405.

32. Cain JM, Zaino R, Shearer D, et al. Expression of a retinol dehydrogenase (hRoDH-4), a member of the retinol/steroid dehydrogenase family implicated in retinoic acid biosynthesis, in normal and neoplastic endometria. Am J Obstet Gynecol $2002 ; 186: 675-83$. 
33. Cerignoli F, Guo X, Cardinali B, et al. retSDR1, a short-chain retinol dehydrogenase/reductase, is retinoic acid-inducible and frequently deleted in human neuroblastoma cell lines. Cancer Res $2002 ; 62$ : 1196-204.

34. Martras S, Alvarez R, Martinez SE, et al. The specificity of alcohol dehydrogenase with cis-retinoids. Activity with 11-cis-retinol and localization in retina. Eur J Biochem $2004 ; 271: 1660-70$.

35. Deltour L, Foglio MH, Duester G. Metabolic deficiencies in alcohol dehydrogenase Adh1, Adh3, and Adh4 null mutant mice. Overlapping roles of Adhl and Adh4 in ethanol clearance and metabolism of retinol to retinoic acid. J Biol Chem $1999 ; 274$ : 16796-801.

36. Molotkov A, Fan X, Deltour $L$, et al. Stimulation of retinoic acid production and growth by ubiquitously expressed alcohol dehydrogenase Adh3. Proc Natl Acad Sci USA 2002 ; 99 : 5337-42.

37. Lee SL, Wang MF, Lee AI, Yin SJ. The metabolic role of human ADH3 functioning as ethanol dehydrogenase. FEBS Lett $2003 ; 544: 143-7$.

38. Molotkov A, Fan X, Duester G. Excessive vitamin A toxicity in mice genetically deficient in either alcohol dehydrogenase Adh1 or Adh3. Eur J Biochem 2002; 269:2607-12.

39. Boleda MD, Saubi N, Farres J, Pares X. Physiological substrates for rat alcohol dehydrogenase classes: aldehydes of lipid peroxidation, omega-hydroxyfatty acids, and retinoids. Arch Biochem Biophys 1993; $307: 85-90$.

40. Allali-Hassani A, Peralba JM, Martras S, et al. Retinoids, omega-hydroxyfatty acids and cytotoxic aldehydes as physiological substrates, and $\mathrm{H} 2$-receptor antagonists as pharmacological inhibitors, of human class IV alcohol dehydrogenase. FEBS Lett $1998 ; 426: 362-6$.

41. Labrecque J, Bhat PV, Lacroix A. Purification and partial characterization of a rat kidney aldehyde dehydrogenase that oxidizes retinal to retinoic acid. Biochem Cell Biol 1993; $71: 85-9$.

42. Posch KC, Burns RD, Napoli JL. Biosynthesis of all-trans-retinoic acid from retinal. Recognition of retinal bound to cellular retinol binding protein (type I) as substrate by a purified cytosolic dehydrogenase. J Biol Chem 1992 ; 267 : 19676-82.

43. El Akawi Z, Napoli JL. Rat liver cytosolic retinal dehydrogenase: comparison of 13-cis-, 9-cis-, and all-trans-retinal as substrates and effects of cellular retinoid-binding proteins and retinoic acid on activity. Biochemistry $1994 ; 33: 1938-43$

44. Gagnon I, Duester G, Bhat PV. Enzymatic characterization of recombinant mouse retinal dehydrogenase type 1. Biochem Pharmacol 2003; 65: 1685-90.

45. Gagnon I, Duester G, Bhat PV. Kinetic analysis of mouse retinal dehydrogenase type-2 (RALDH2) for retinal substrates. Biochim Biophys Acta 2002 ; 1596 : 156-62.

46. Grun F, Hirose Y, Kawauchi S, et al. Aldehyde dehydrogenase 6, a cytosolic retinaldehyde dehydrogenase prominently expressed in sensory neuroepithelia during development. J Biol Chem $2000 ; 275: 41210-8$

47. Lin $M$, Zhang $M$, Abraham $M$, et al. Mouse retinal dehydrogenase 4 (RALDH4), molecular cloning, cellular expression, and activity in 9-cis-retinoic acid biosynthesis in intact cells. J Biol Chem $2003 ; 278: 9856-61$

48. Lin M, Napoli JL. cDNA cloning and expression of a human aldehyde dehydrogenase (ALDH) active with 9-cis-retinal and identification of a rat ortholog, ALDH12.J Biol Chem $2000 ; 275$ : 40106-12.

49. Sonneveld $\varepsilon$, van den Brink $C \varepsilon$, van der Leede BM, et al. Human retinoic acid (RA) 4-hydroxylase (CYP26) is highly specific for all-trans-RA and can be induced through RA receptors in human breast and colon carcinoma cells. Cell Growth Differ $1998 ; 9: 629-37$.

50. Marikar Y, Wang Z, Duell EA, et al. Retinoic acid receptors regulate expression of retinoic acid 4hydroxylase that specifically inactivates all-trans retinoic acid in human keratinocyte $\mathrm{HaCaT}$ cells. J Invest Dermatol $1998 ; 111: 434-9$.

51. Taimi M, Helvig C, Wisniewski J, et al. A novel human cytochrome P450, CyP26Cl, involved in metabolism of 9-cis and all-trans isomers of retinoic acid. J Biol Chem $2004 ; 279: 77-85$.
52. Ong DE. A novel retinol-binding protein from rat. Purification and partial characterization. J Biol Chem $1984 ; 259$ : 1476-82.

53. MacDonald PN, Ong DE. Binding specificities of cellular retinol-binding protein and cellular retinol-binding protein, type II. J Biol Chem 1987; $262: 10550-6$.

54. Newcomer ME. Retinoid-binding proteins: structural determinants important for function. FASEB J $1995 ; 9: 229-39$.

55. Inagami $S, 0 n g$ DE. Purification and partial characterization of cellular retinol-binding protein, type two, from human small intestine. J Nutr $1992 ; 122: 450-6$.

56. MacDonald PN, Ong DE. Binding specificities of cellular retinol-binding protein and cellular retinol-binding protein, type II. J Biol Chem 1987 ; $262: 10550-6$.

57. Fiorella PD, Napoli JL. Expression of cellular retinoic acid binding protein (CRABP) in Escherichia coli. Characterization and evidence that holoCRABP is a substrate in retinoic acid metabolism. J Biol Chem 1991; 266: 16572-9.

58. Fogh K, Voorhees JJ, Astrom A. Expression, purification, and binding properties of human cellular retinoic acid-binding protein type I and type II. Arch Biochem Biophys 1993 ; 300 : 751-5.

59. Allenby G, Bocquel MT, Saunders M, et al. Retinoic acid receptors and retinoid $X$ receptors: interactions with endogenous retinoic acids. Proc Natl Acad Sci USA 1993; $90: 30-4$.

60. Kleywegt GJ, Bergfors T, Senn H, et al. Crystal structures of cellular retinoic acid binding proteins I and II in complex with all-trans-retinoic acid and a synthetic retinoid. Structure $1994 ; 2$ : 1241-58.

61. Fiorella PD, Giguere V, Napoli JL. Expression of cellular retinoic acidbinding protein (type II) in Escherichia coli. Characterization and comparison to cellular retinoic acid-binding protein (type I). J Biol Chem $1993 ; 268: 21545-52$

62. Norris AW, Cheng L, Giguere V, et al. Measurement of subnanomolar retinoic acid binding affinities for cellular retinoic acid binding proteins by fluorometric titration. Biochim Biophys Acta $1994 ; 1209$ : 10-8.

63. Petkovich M, Brand NJ, Krust A, Chambon P. A human retinoic acid receptor which belongs to the family of nuclear receptors. Nature 1987 ; $330: 444-50$

64. Levin AA, Sturzenbecker LJ, Kazmer S, et al. 9-cis retinoic acid stereoisomer binds and activates the nuclear receptor RXR alpha. Nature $1992 ; 355: 359-61$

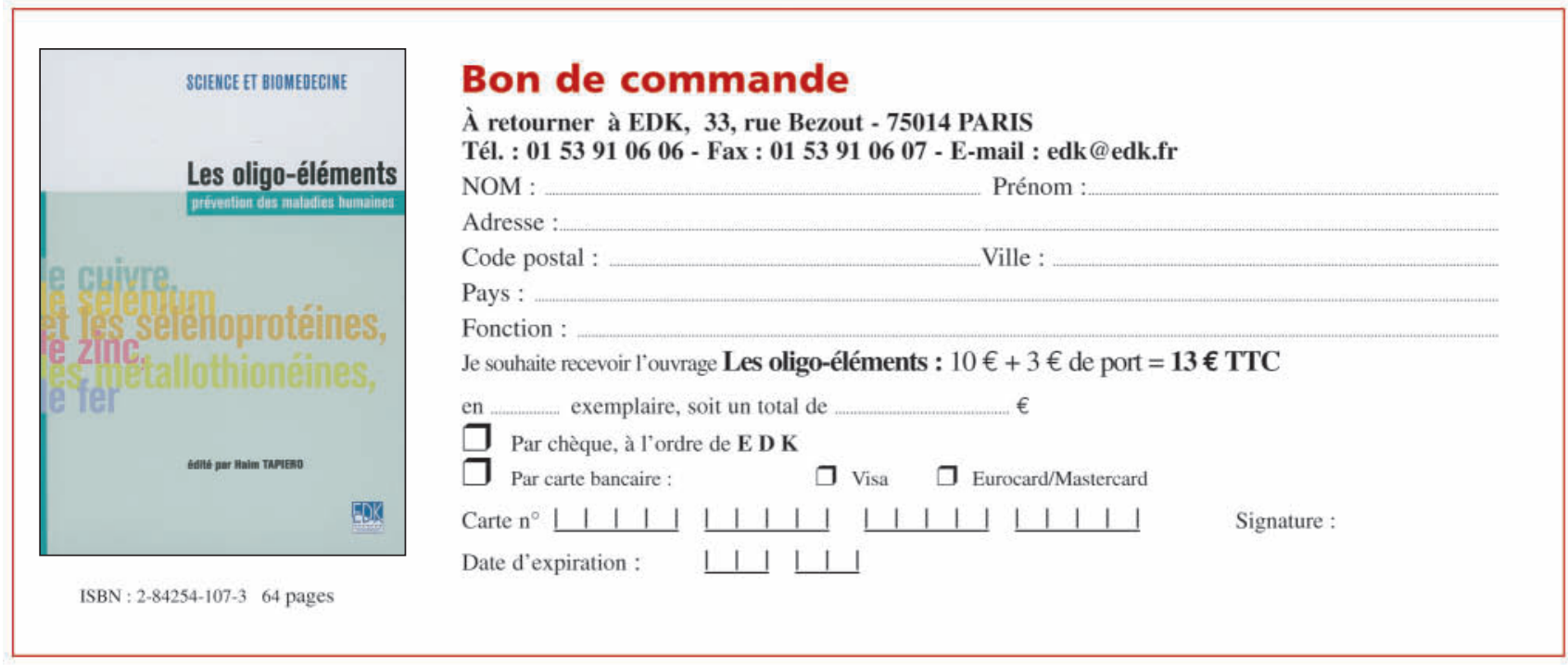

